\title{
Some new Hermite-Hadamard type inequalities for $s$-convex functions and their applications
}

Serap Özcan ${ }^{1 *}$ (D) and Imdat Işcan²

"Correspondence:

serapozcann@yahoo.com; serapozcan@klu.edu.tr

'Department of Mathematics, Faculty of Art and Science, Kırklareli University, Kırklareli, Turkey Full list of author information is available at the end of the article

\begin{abstract}
In this paper, we establish some new integral inequalities of Hermite-Hadamard type for s-convex functions by using the Hölder-işcan integral inequality. We also compare our new results with the known results and show that the results which we obtained are better than the known results. Finally, we give some applications to trapezoidal formula and to special means.
\end{abstract}

MSC: 26D15; 26A51

Keywords: Convex function; s-convex function; Hermite-Hadamard type inequality; Hölder inequality; Hölder-Işcan inequality; Integral inequalities; Trapezoidal formula; Special means

\section{Introduction}

The classical or the usual convexity is defined as follows:

A function $f: I \subseteq \mathbb{R} \rightarrow \mathbb{R}$ is said to be convex on interval $I$ if

$$
f(t x+(1-t) y) \leq t f(x)+(1-t) f(y)
$$

for all $x, y \in I$ and $t \in[0,1]$.

Let $f: I \subseteq \mathbb{R} \longrightarrow \mathbb{R}$ be a convex function on $I$ and $a, b \in I$ with $a<b$. The following inequality is known in the literature as the Hermite-Hadamard inequality for convex functions:

$$
f\left(\frac{a+b}{2}\right) \leq \frac{1}{b-a} \int_{a}^{b} f(x) d x \leq \frac{f(a)+f(b)}{2} .
$$

In [8], Hudzik and Maligranda considered the class of $s$-convex functions in second sense. This is defined as follows:

A function $f:[0, \infty) \rightarrow \mathbb{R}$ is said to be $s$-convex in second sense if the inequality

$$
f(t x+(1-t) y) \leq t^{s} f(x)+(1-t)^{s} f(y)
$$

holds for all $x, y \in[0, \infty), t \in[0,1]$, and $s \in(0,1]$.

(c) The Author(s) 2019. This article is distributed under the terms of the Creative Commons Attribution 4.0 International License (http://creativecommons.org/licenses/by/4.0/), which permits unrestricted use, distribution, and reproduction in any medium, provided you give appropriate credit to the original author(s) and the source, provide a link to the Creative Commons license, and indicate if changes were made. 
In [4], Dragomir and Fitzpatrick established a variant of Hermite-Hadamard inequality which holds for the $s$-convex functions.

Theorem 1.1 Suppose that $f:[0, \infty) \rightarrow[0, \infty)$ is an s-convex function in second sense, where $\in(0,1]$. Let $a, b \in[0, \infty)$ and $a<b$.Iff $\in L[0,1]$, then the following inequality holds:

$$
2^{s-1} f\left(\frac{a+b}{2}\right) \leq \frac{1}{b-a} \int_{a}^{b} f(x) d x \leq \frac{f(a)+f(b)}{s+1} .
$$

For the generalizations and applications of the Hermite-Hadamard inequalities, see [1$3,5-7,9,12-15,17,19-21]$.

A number of studies have shown that many of the results obtained about the theory of inequalities has a close relationship with the theory of convex functions.

The celebrated inequality of Hölder is well known for its fundamental role in many branches of pure and applied sciences. It has also important applications to the theory of convex functions as well as in many disciplines of applied mathematics. The powermean integral inequality is also one of the most famous inequalities on applications to convex functions.

Theorem 1.2 (Hölder inequality for integrals [16]) Let $p>1$ and $1 / p+1 / q=1$. Iff and $g$ are real functions defined on $[a, b]$ and if $|f|^{p}$ and $|g|^{q}$ are integrable on $[a, b]$, then

$$
\int_{a}^{b}|f(x) g(x)| d x \leq\left(\int_{a}^{b}|f(x)|^{p} d x\right)^{\frac{1}{p}}\left(\int_{a}^{b}|g(x)|^{q} d x\right)^{\frac{1}{q}},
$$

with equality holds if and only if $A|f(x)|^{p}=B|g(x)|^{q}$ almost everywhere, where $A$ and $B$ are constants.

A different version of Hölder integral inequality is given as follows.

Theorem 1.3 (Power-mean integral inequality) Let $q \geq 1$. If $f$ and $g$ are real functions defined on $[a, b]$ and if $|f|,|f||g|^{q}$ are integrable on $[a, b]$, then

$$
\int_{a}^{b}|f(x) g(x)| d x \leq\left(\int_{a}^{b}|f(x)| d x\right)^{1-\frac{1}{q}}\left(\int_{a}^{b}|f(x)||g(x)|^{q} d x\right)^{\frac{1}{q}} .
$$

In [10], İşcan obtained the following inequality for integrals which gives better results than the classical Hölder inequality.

Theorem 1.4 (Hölder-İscan integral inequality) Let $p>1$ and $1 / p+1 / q=1$. Iff and $g$ are real functions defined on $[a, b]$ and if $|f|^{p}$ and $|g|^{q}$ are integrable on $[a, b]$, then

$$
\begin{aligned}
\int_{a}^{b}|f(x) g(x)| d x \leq & \frac{1}{b-a}\left\{\left(\int_{a}^{b}(b-x)|f(x)|^{p} d x\right)^{\frac{1}{p}}\left(\int_{a}^{b}(b-x)|g(x)|^{q} d x\right)^{\frac{1}{q}}\right. \\
& \left.+\left(\int_{a}^{b}(x-a)|f(x)|^{p} d x\right)^{\frac{1}{p}}\left(\int_{a}^{b}(x-a)|g(x)|^{q} d x\right)^{\frac{1}{q}}\right\} \\
\leq & \left(\int_{a}^{b}|f(x)|^{p} d x\right)^{\frac{1}{p}}\left(\int_{a}^{b}|g(x)|^{q} d x\right)^{\frac{1}{q}} .
\end{aligned}
$$


In [11], a different representation of Hölder-İşcan inequality was given as follows.

Theorem 1.5 (Improved power-mean integral inequality) Let $q \geq 1$. If $f$ and $g$ are real functions defined on $[a, b]$ and if $|f|,|f||g|^{q}$ are integrable functions on $[a, b]$, then

$$
\begin{aligned}
\int_{a}^{b}|f(x) g(x)| d x \leq & \frac{1}{b-a}\left\{\left(\int_{a}^{b}(b-x)|f(x)| d x\right)^{1-\frac{1}{q}}\left(\int_{a}^{b}(b-x)|f(x)||g(x)|^{q} d x\right)^{\frac{1}{q}}\right. \\
& \left.+\left(\int_{a}^{b}(x-a)|f(x)| d x\right)^{1-\frac{1}{q}}\left(\int_{a}^{b}(x-a)|f(x)||g(x)|^{q} d x\right)^{\frac{1}{q}}\right\} \\
\leq & \left(\int_{a}^{b}|f(x)| d x\right)^{1-\frac{1}{q}}\left(\int_{a}^{b}|f(x)||g(x)|^{q} d x\right)^{\frac{1}{q}} .
\end{aligned}
$$

\section{Main results}

In this section we obtain some new results about Hermite-Hadamard inequality for $s$ convex functions by using Hölder-İscan integral inequality and improved power-mean integral inequality which provide better approach than the classical Hölder and powermean integral inequalities, respectively.

In [5], Dragomir and Pearce obtained the following equality for differentiable functions.

Lemma 2.1 Let $f: I \subseteq \mathbb{R} \longrightarrow \mathbb{R}$ be a differentiable function on $I^{\circ}$ where $a, b \in I$ with $a<b$. If $f^{\prime} \in L[a, b]$, then the following equality holds:

$$
\frac{f(a)+f(b)}{2}-\frac{1}{b-a} \int_{a}^{b} f(x) d x=\frac{b-a}{2} \int_{0}^{1}(1-2 t) f^{\prime}(t a+(1-t) b) d t .
$$

In [17], Muddassar et al. obtained the following inequality for $s$-convex functions by using Lemma 2.1 and Hölder integral inequality.

Theorem 2.1 Letf $: I^{\circ} \subseteq \mathbb{R} \rightarrow \mathbb{R}$ be a differentiable function on $I^{\circ}, a, b \in I^{\circ}$ with $a<b$ and $f^{\prime} \in L[a, b]$. If $\left|f^{\prime}\right|^{q}$ is s-convex on $[a, b], p>1$ such that $q=\frac{p}{p-1}$, then

$$
\left|\frac{f(a)+f(b)}{2}-\frac{1}{b-a} \int_{a}^{b} f(x) d x\right| \leq \frac{b-a}{2(p+1)^{\frac{1}{p}}}\left(\frac{\left|f^{\prime}(a)\right|^{q}+\left|f^{\prime}(b)\right|^{q}}{s+1}\right)^{\frac{1}{q}} .
$$

In the following theorem, we will obtain a new upper bound for the right-hand side of Hermite-Hadamard inequality for $s$-convex functions, which is better than inequality (3).

Theorem 2.2 Letf $: I^{\circ} \subseteq \mathbb{R} \rightarrow \mathbb{R}$ be a differentiable function on $I^{\circ}, a, b \in I^{\circ}$ with $a<b$ and $f^{\prime} \in L[a, b]$. If $\left|f^{\prime}\right|^{q}$ is s-convex on $[a, b]$, where $p>1$ such that $q=\frac{p}{p-1}$, then

$$
\begin{aligned}
& \left|\frac{f(a)+f(b)}{2}-\frac{1}{b-a} \int_{a}^{b} f(x) d x\right| \\
& \leq \frac{b-a}{2^{\frac{p+1}{p}}(p+1)^{\frac{1}{p}}} \cdot \frac{1}{(s+1)^{\frac{1}{q}}} \\
& \quad \times\left\{\left[\frac{(s+1)\left|f^{\prime}(a)\right|^{q}+\left|f^{\prime}(b)\right|^{q}}{s+2}\right]^{\frac{1}{q}}+\left[\frac{\left|f^{\prime}(a)\right|^{q}+(s+1)\left|f^{\prime}(b)\right|^{q}}{s+2}\right]^{\frac{1}{q}}\right\} .
\end{aligned}
$$


Proof From Lemma 2.1 and using Hölder-İşcan integral inequality (1), we have

$$
\begin{aligned}
& \left|\frac{f(a)+f(b)}{2}-\frac{1}{b-a} \int_{a}^{b} f(x) d x\right| \\
& \leq \frac{b-a}{2} \int_{0}^{1}|1-2 t|\left|f^{\prime}(t a+(1-t) b)\right| d t \\
& \leq \frac{b-a}{2}\left\{\left(\int_{0}^{1}(1-t)|1-2 t|^{p} d t\right)^{\frac{1}{p}}\left(\int_{0}^{1}(1-t)\left|f^{\prime}(t a+(1-t) b)\right|^{q} d t\right)^{\frac{1}{q}}\right. \\
& \left.\quad+\left(\int_{0}^{1} t|1-2 t|^{p} d t\right)^{\frac{1}{p}}\left(\int_{0}^{1} t\left|f^{\prime}(t a+(1-t) b)\right|^{q} d t\right)^{\frac{1}{q}}\right\} .
\end{aligned}
$$

Since $\left|f^{\prime}\right|^{q}$ is $s$-convex on $[a, b]$, then

$$
\begin{aligned}
\int_{0}^{1} t\left|f^{\prime}(t a+(1-t) b)\right|^{q} d t & \leq \int_{0}^{1} t\left[t^{s}\left|f^{\prime}(a)\right|^{q}+(1-t)^{s}\left|f^{\prime}(b)\right|^{q}\right] d t \\
& =\frac{(s+1)\left|f^{\prime}(a)\right|^{q}+\left|f^{\prime}(b)\right|^{q}}{(s+1)(s+2)}
\end{aligned}
$$

and

$$
\begin{aligned}
\int_{0}^{1}(1-t)\left|f^{\prime}(t a+(1-t) b)\right|^{q} d t & =\int_{0}^{1} t\left|f^{\prime}(t b+(1-t) a)\right|^{q} d t \\
& =\frac{\left|f^{\prime}(a)\right|^{q}+(s+1)\left|f^{\prime}(b)\right|^{q}}{(s+1)(s+2)}
\end{aligned}
$$

and also

$$
\begin{aligned}
\int_{0}^{1} t|1-2 t|^{p} d t & =\int_{0}^{1}(1-t)|1-2 t|^{p} d t \\
& =\frac{1}{2(p+1)} .
\end{aligned}
$$

By inequalities (5), (6), and (7), we get inequality (4).

Corollary 2.1 Let $f: I^{\circ} \subseteq \mathbb{R} \rightarrow \mathbb{R}$ be a differentiable function on $I^{\circ}, a, b \in I^{\circ}$ with $a<b$ and $f^{\prime} \in L[a, b]$. If $\left|f^{\prime}\right|^{q}$ is s-convex on $[a, b]$, where $p>1$ such that $q=\frac{p}{p-1}$, then

$$
\begin{aligned}
& \left|\frac{f(a)+f(b)}{2}-\frac{1}{b-a} \int_{a}^{b} f(x) d x\right| \\
& \quad \leq \frac{b-a}{2^{\frac{p+1}{p}}(p+1)^{\frac{1}{p}}} \frac{1}{(s+2)^{\frac{1}{q}}}\left(1+\frac{1}{(s+1)^{\frac{1}{q}}}\right)\left(\left|f^{\prime}(a)\right|+\left|f^{\prime}(b)\right|\right) .
\end{aligned}
$$

Proof Using the fact $\sum_{i=1}^{n}\left(a_{i}+b_{i}\right)^{k} \leq \sum\left(a_{i}\right)^{k}+\sum\left(b_{i}\right)^{k}$ for $k \in(0,1)$ with $p>1$ such that $q=\frac{p}{p-1}$ completes the proof. 
Remark 2.1 Inequality (4) is better than inequality (3). Indeed, since the function $g$ : $[0, \infty) \rightarrow \mathbb{R}, g(x)=x^{r}, r \in(0,1]$ is concave, we can write

$$
\frac{\alpha^{r}+\beta^{r}}{2}=\frac{g(\alpha)+g(\beta)}{2} \leq g\left(\frac{\alpha+\beta}{2}\right)=\left(\frac{\alpha+\beta}{2}\right)^{r}
$$

for all $\alpha, \beta \geq 0$. In inequality (9), if we choose

$$
\alpha=\frac{(s+1)\left|f^{\prime}(a)\right|^{q}+\left|f^{\prime}(b)\right|^{q}}{s+2}, \quad \beta=\frac{\left|f^{\prime}(a)\right|^{q}+(s+1)\left|f^{\prime}(b)\right|^{q}}{s+2}
$$

and $r=\frac{1}{q}$, then we have

$$
\begin{aligned}
& \frac{1}{2}\left[\frac{(s+1)\left|f^{\prime}(a)\right|^{q}+\left|f^{\prime}(b)\right|^{q}}{s+2}\right]^{\frac{1}{q}}+\frac{1}{2}\left[\frac{\left|f^{\prime}(a)\right|^{q}+(s+1)\left|f^{\prime}(b)\right|^{q}}{s+2}\right]^{\frac{1}{q}} \\
& \quad \leq\left[\frac{\left|f^{\prime}(a)\right|^{q}+\left|f^{\prime}(b)\right|^{q}}{2}\right]^{\frac{1}{q}} .
\end{aligned}
$$

Thus, we obtain the following inequality:

$$
\begin{aligned}
& \frac{b-a}{2^{\frac{p+1}{p}}(p+1)^{\frac{1}{p}}} \cdot \frac{1}{(s+1)^{\frac{1}{q}}} \\
& \quad \times\left\{\left[\frac{(s+1)\left|f^{\prime}(a)\right|^{q}+\left|f^{\prime}(b)\right|^{q}}{s+2}\right]^{\frac{1}{q}}+\left[\frac{\left|f^{\prime}(a)\right|^{q}+(s+1)\left|f^{\prime}(b)\right|^{q}}{s+2}\right]^{\frac{1}{q}}\right\} \\
& \leq \frac{b-a}{2^{\frac{1}{p}}(p+1)^{\frac{1}{p}}} \cdot \frac{1}{(s+1)^{\frac{1}{q}}}\left[\frac{\left.f^{\prime}(a)\right|^{q}+\left|f^{\prime}(b)\right|^{q}}{2}\right]^{\frac{1}{q}} \\
& =\frac{b-a}{2(p+1)^{\frac{1}{p}}} \cdot \frac{1}{(s+1)^{\frac{1}{q}}}\left(\left|f^{\prime}(a)\right|^{q}+\left|f^{\prime}(b)\right|^{q}\right)^{\frac{1}{q}} .
\end{aligned}
$$

If we take $s=1$ in Remark 2.1, we have the following result which was obtained by İșcan in [10].

Remark 2.2 Let $f: I^{\circ} \subseteq \mathbb{R} \rightarrow \mathbb{R}$ be a differentiable function on $I^{\circ}, a, b \in I^{\circ}$ with $a<b$ and $f^{\prime} \in L[a, b]$. If $\left|f^{\prime}\right|^{q}$ is convex on $[a, b]$, then

$$
\begin{aligned}
& \left|\frac{f(a)+f(b)}{2}-\frac{1}{b-a} \int_{a}^{b} f(x) d x\right| \\
& \quad \leq \frac{b-a}{4(p+1)^{\frac{1}{p}}}\left\{\left[\frac{2\left|f^{\prime}(a)\right|^{q}+\left|f^{\prime}(b)\right|^{q}}{3}\right]^{\frac{1}{q}}+\left[\frac{\left|f^{\prime}(a)\right|^{q}+2\left|f^{\prime}(b)\right|^{q}}{3}\right]^{\frac{1}{q}}\right\} \\
& \quad \leq \frac{b-a}{2(p+1)^{\frac{1}{p}}}\left[\frac{\left|f^{\prime}(a)\right|^{q}+\left|f^{\prime}(b)\right|^{q}}{2}\right]^{\frac{1}{q}},
\end{aligned}
$$

where $\frac{1}{p}+\frac{1}{q}=1$.

In [17], Muddassar et al. obtained the following inequality by using Lemma 2.1 and power-mean integral inequality. 
Theorem 2.3 Let $f: I^{\circ} \subseteq \mathbb{R} \longrightarrow \mathbb{R}$ be a differentiable function on $I^{\circ}$ where $a, b \in I$ with $a<b$ and $f^{\prime} \in L[a, b]$. If $\left|f^{\prime}\right|^{q}$ is s-convex on $[a, b]$ for some $p>1$ such that $q=\frac{p}{p-1}$, then the following inequality holds:

$$
\begin{aligned}
& \left|\frac{f(a)+f(b)}{2}-\frac{1}{b-a} \int_{a}^{b} f(x) d x\right| \\
& \quad \leq \frac{b-a}{2^{\frac{p+1}{p}}}\left[\left(\frac{s+2^{-s}}{(s+1)(s+2)}\right)\left(\left|f^{\prime}(a)\right|^{q}+\left|f^{\prime}(b)\right|^{q}\right)\right]^{\frac{1}{q}} .
\end{aligned}
$$

If Theorem 2.3 is proved again by using improved power-mean integral inequality, then we get the following result.

Theorem 2.4 Let $f: I^{\circ} \subseteq \mathbb{R} \longrightarrow \mathbb{R}$ be a differentiable function on $I^{\circ}$ where $a, b \in I$ with $a<b$ and $f^{\prime} \in L[a, b]$. If $\left|f^{\prime}\right|^{q}$ is s-convex on $[a, b]$ for some $p>1$ such that $q=\frac{p}{p-1}$, then the following inequality holds:

$$
\begin{aligned}
& \left|\frac{f(a)+f(b)}{2}-\frac{1}{b-a} \int_{a}^{b} f(x) d x\right| \\
& \leq \frac{b-a}{2 \cdot 4^{\frac{1}{p}}} \cdot \frac{1}{(s+1)^{\frac{1}{q}}} \\
& \quad \times\left\{\left[\frac{(s+1)\left(s+1+2^{-s-1}\right)\left|f^{\prime}(a)\right|^{q}+\left(s-1+2^{-s-1}(s+5)\right)\left|f^{\prime}(b)\right|^{q}}{(s+2)(s+3)}\right]^{\frac{1}{q}}\right. \\
& \left.\quad+\left[\frac{\left(s-1+2^{-s-1}(s+5)\right)\left|f^{\prime}(a)\right|^{q}+(s+1)\left(s+1+2^{-s-1}\right)\left|f^{\prime}(b)\right|^{q}}{(s+2)(s+3)}\right]^{\frac{1}{q}}\right\} .
\end{aligned}
$$

Proof From Lemma 2.1 and applying improved power-mean integral inequality (2) for $q>1$, we have

$$
\begin{aligned}
& \left|\frac{f(a)+f(b)}{2}-\frac{1}{b-a} \int_{a}^{b} f(x) d x\right| \\
& \quad \leq \frac{b-a}{2}\left\{\left(\int_{0}^{1}(1-t)|1-2 t| d t\right)^{\frac{1}{p}}\left(\int_{0}^{1}(1-t)|1-2 t|\left|f^{\prime}(t a+(1-t) b)\right|^{q} d t\right)^{\frac{1}{q}}\right. \\
& \left.\quad+\left(\int_{0}^{1} t|1-2 t| d t\right)^{\frac{1}{p}}\left(\int_{0}^{1} t|1-2 t|\left|f^{\prime}(t a+(1-t) b)\right|^{q} d t\right)^{\frac{1}{q}}\right\}
\end{aligned}
$$

By s-convexity of $\left|f^{\prime}\right|^{q}$ on $[a, b]$, we have

$$
\begin{aligned}
& \int_{0}^{1} t|1-2 t|\left|f^{\prime}(t a+(1-t) b)\right|^{q} d t \\
& \quad \leq \int_{0}^{1} t|1-2 t|\left[t^{s}\left|f^{\prime}(a)\right|^{q}+(1-t)^{s}\left|f^{\prime}(b)\right|^{q}\right] d t \\
& \quad=\frac{(s+1)\left(s+1+2^{-s-1}\right)\left|f^{\prime}(a)\right|^{q}+\left(s-1+2^{-s-1}(s+5)\right)\left|f^{\prime}(b)\right|^{q}}{(s+1)(s+2)(s+3)}
\end{aligned}
$$


and

$$
\begin{aligned}
& \int_{0}^{1}(1-t)|1-2 t|\left|f^{\prime}(t a+(1-t) b)\right|^{q} d t \\
& \quad=\int_{0}^{1} t|1-2 t|\left|f^{\prime}(t b+(1-t) a)\right|^{q} d t \\
& \quad \leq \frac{\left(s-1+2^{-s-1}(s+5)\right)\left|f^{\prime}(a)\right|^{q}+(s+1)\left(s+1+2^{-s-1}\right)\left|f^{\prime}(b)\right|^{q}}{(s+1)(s+2)(s+3)}
\end{aligned}
$$

Furthermore,

$$
\begin{aligned}
\int_{0}^{1} t|1-2 t| d t & =\int_{0}^{1}(1-t)|1-2 t| d t \\
& =\frac{1}{4}
\end{aligned}
$$

By using inequalities (12) and (13), we get inequality (11).

Remark 2.3 Inequality (11) is better than inequality (10). Indeed, using inequality (9) in Remark 2.1, we have

$$
\begin{aligned}
& \frac{1}{2}\left[\frac{(s+1)\left(s+1+2^{-s-1}\right)\left|f^{\prime}(a)\right|^{q}+\left(s-1+2^{-s-1}(s+5)\right)\left|f^{\prime}(b)\right|^{q}}{(s+2)(s+3)}\right]^{\frac{1}{q}} \\
& \quad+\frac{1}{2}\left[\frac{\left(s-1+2^{-s-1}(s+5)\right)\left|f^{\prime}(a)\right|^{q}+(s+1)\left(s+1+2^{-s-1}\right)\left|f^{\prime}(b)\right|^{q}}{(s+2)(s+3)}\right]^{\frac{1}{q}} \\
& \leq\left[\left(\frac{s+2^{-s}}{s+2}\right)\left(\frac{\left|f^{\prime}(a)\right|^{q}+\left|f^{\prime}(b)\right|^{q}}{2}\right)\right]^{\frac{1}{q}} .
\end{aligned}
$$

Therefore, we obtain

$$
\begin{aligned}
\frac{b-a}{2.4^{\frac{1}{p}}} & \cdot \frac{1}{(s+1)^{\frac{1}{q}}} \\
& \times\left\{\left[\frac{(s+1)\left(s+1+2^{-s-1}\right)\left|f^{\prime}(a)\right|^{q}+\left(s-1+2^{-s-1}(s+5)\right)\left|f^{\prime}(b)\right|^{q}}{(s+2)(s+3)}\right]^{\frac{1}{q}}\right. \\
& \left.+\left[\frac{\left(s-1+2^{-s-1}(s+5)\right)\left|f^{\prime}(a)\right|^{q}+(s+1)\left(s+1+2^{-s-1}\right)\left|f^{\prime}(b)\right|^{q}}{(s+2)(s+3)}\right]^{\frac{1}{q}}\right\} \\
\leq & \frac{b-a}{4^{\frac{1}{p}}} \cdot \frac{1}{(s+1)^{\frac{1}{q}}}\left[\left(\frac{s+2^{-s}}{s+2}\right)\left(\frac{\left|f^{\prime}(a)\right|^{q}+\left|f^{\prime}(b)\right|^{q}}{2}\right)\right]^{\frac{1}{q}} \\
= & \frac{b-a}{2^{\frac{p+1}{p}}}\left[\left(\frac{s+2^{-s}}{(s+1)(s+2)}\right)\left(\left|f^{\prime}(a)\right|^{q}+\left|f^{\prime}(b)\right|^{q}\right)\right]^{\frac{1}{q}} \cdot
\end{aligned}
$$

If we take $s=1$ in Remark 2.3, we have the following result for convex functions which is better than the inequality given in [18, Theorem 1$]$. 
Remark 2.4 Let $f: I^{\circ} \subseteq \mathbb{R} \rightarrow \mathbb{R}$ be a differentiable function on $I^{\circ}, a, b \in I^{\circ}$ with $a<b$ and $f^{\prime} \in L[a, b]$. If $\left|f^{\prime}\right|^{q}$ is convex on $[a, b]$, then

$$
\begin{aligned}
& \left|\frac{f(a)+f(b)}{2}-\frac{1}{b-a} \int_{a}^{b} f(x) d x\right| \\
& \quad \leq \frac{b-a}{4.2^{\frac{1}{p}}}\left\{\left[\frac{3\left|f^{\prime}(a)\right|^{q}+\left|f^{\prime}(b)\right|^{q}}{4}\right]^{\frac{1}{q}}+\left[\frac{\left|f^{\prime}(a)\right|^{q}+3\left|f^{\prime}(b)\right|^{q}}{4}\right]^{\frac{1}{q}}\right\} \\
& \quad \leq \frac{b-a}{4}\left[\frac{\left.f^{\prime}(a)\right|^{q}+\left|f^{\prime}(b)\right|^{q}}{2}\right]^{\frac{1}{q}},
\end{aligned}
$$

where $\frac{1}{p}+\frac{1}{q}=1$.

\section{An application to trapezoidal formula}

Let $D$ be a division of the interval $[a, b]$, i.e., $D: a=x_{0}<x_{1}<\cdots<x_{n-1}<x_{n}=b$ and consider the quadrature formula

$$
I=\int_{a}^{b} f(x) d x=T(f, D)+R(f, D)
$$

where

$$
T(f, D)=\sum_{k=0}^{n-1} \frac{f\left(x_{k}\right)+f\left(x_{k+1}\right)}{2}\left(x_{k+1}-x_{k}\right)
$$

is the trapezoidal formula and $R(f, D)$ denotes the associated approximation error of the integral $I$.

Proposition 3.1 Let $f: I \subseteq \mathbb{R} \rightarrow \mathbb{R}$ be a differentiable function on $I^{\circ}, a, b \in I$ with $a<b$ and $f^{\prime} \in L[a, b]$. If $\left|f^{\prime}\right|^{q}$ is s-convex on $[a, b]$ for $p, q>1$, for every division $D$ of $[a, b]$, the following trapezoidal error estimate holds:

$$
\begin{aligned}
|R(f, D)| \leq & \frac{1}{2^{\frac{p+1}{p}}(p+1)^{\frac{1}{p}}} \cdot \frac{1}{(s+2)^{\frac{1}{q}}}\left(1+\frac{1}{(s+1)^{\frac{1}{q}}}\right) \\
& \times \sum_{k=0}^{n-1}\left(x_{k+1}-x_{k}\right)^{2}\left(\left|f^{\prime}\left(x_{k}\right)\right|+\left|f^{\prime}\left(x_{k+1}\right)\right|\right) .
\end{aligned}
$$

Proof Applying Corollary 2.1 on the subintervals $\left[x_{k}, x_{k+1}\right],(k=0,1,2, \ldots, n-1)$ of the division $D$, we have

$$
\begin{aligned}
& \left|\frac{f\left(x_{k}\right)+f\left(x_{k+1}\right)}{2}-\frac{1}{x_{k+1}-x_{k}} \int_{x_{k}}^{x_{k+1}} f(x) d x\right| \\
& \quad \leq \frac{x_{k+1}-x_{k}}{2^{\frac{p+1}{p}}(p+1)^{\frac{1}{p}}} \cdot \frac{1}{(s+2)^{\frac{1}{q}}}\left(1+\frac{1}{(s+1)^{\frac{1}{q}}}\right)\left(\left|f^{\prime}\left(x_{k}\right)\right|+\left|f^{\prime}\left(x_{k+1}\right)\right|\right) .
\end{aligned}
$$


Summing over $k$ from 0 to $n-1$ and using the generalized triangle inequality, we get

$$
\begin{aligned}
|R(f, D)| & =\left|T(f, D)-\int_{a}^{b} f(x) d x\right| \\
& =\left|\sum_{k=0}^{n-1}\left(\left(x_{k+1}-x_{k}\right) \frac{f\left(x_{k}\right)+f\left(x_{k+1}\right)}{2}-\int_{x_{k}}^{x_{k+1}} f(x) d x\right)\right| \\
& \leq \sum_{k=0}^{n-1}\left|\left(x_{k+1}-x_{k}\right) \frac{f\left(x_{k}\right)+f\left(x_{k+1}\right)}{2}-\int_{x_{k}}^{x_{k+1}} f(x) d x\right| .
\end{aligned}
$$

So, we have

$$
|R(f, D)| \leq \sum_{k=0}^{n-1}\left(x_{k+1}-x_{k}\right)\left|\frac{f\left(x_{k}\right)+f\left(x_{k+1}\right)}{2}-\frac{1}{x_{k+1}-x_{k}} \int_{x_{k}}^{x_{k+1}} f(x) d x\right|,
$$

which is the required result.

\section{An application to special means}

In [6], Hudzik and Maligranda gave the following example.

Let $a, b, c \in \mathbb{R}$ and $s \in(0,1)$. For $t \in[0, \infty)$, define the function $f:[0, \infty) \rightarrow \mathbb{R}$ as

$$
f(t)= \begin{cases}a, & t=0 \\ b t^{s}+c, & t>0\end{cases}
$$

If $b \geq 0$ and $0 \leq c \leq a$, then $f \in K_{s}^{2}$. So, we have $f:[0,1] \rightarrow[0,1], f(t)=t^{s}, f \in K_{s}^{2}$ for $a=c=0$ and $b=1$.

Let us recall the following special means for arbitrary real numbers $a$ and $b(a \neq b)$.

(1) The arithmetic mean:

$$
A:=A(a, b)=\frac{a+b}{2}
$$

(2) The $p$-logarithmic mean:

$$
L_{p}:=L_{p}(a, b)=\left[\frac{b^{p+1}-a^{p+1}}{(p+1)(b-a)}\right]^{\frac{1}{p}}, \quad p \in \mathbb{R} \backslash\{-1,0\} .
$$

Proposition 4.1 Let $p>1, a<b, s \in(0,1)$, and $q=\frac{p}{p-1}$. Then we have

$$
\begin{aligned}
& \left|A^{s}(a, b)-L_{s}^{s}(a, b)\right| \\
& \quad \leq \frac{b-a}{2^{\frac{p+1}{p}}(p+1)^{\frac{1}{p}}} \cdot \frac{s}{(s+2)^{\frac{1}{q}}}\left(1+\frac{1}{(s+1)^{\frac{1}{q}}}\right)\left(|a|^{s-1}+|b|^{s-1}\right) .
\end{aligned}
$$

Proof By Corollary 2.1 applied for the $s$-convex function $f:[0,1] \rightarrow[0,1], f(x)=x^{s}$ for $s \in(0,1)$, the proof is completed. 


\title{
5 Conclusion
}

In this paper, some new results of Hermite-Hadamard type for $s$-convex functions are established. It is shown that the results obtained here are better than the known results. Some applications of these results to trapezoidal formula and to special means have also been presented. The results of this paper may stimulate further research for the researchers working in this field.

\author{
Acknowledgements \\ Authors are thankful to the editor and anonymous referees for their valuable comments and suggestions. \\ Funding \\ There is no funding for this research article. \\ Availability of data and materials \\ Not applicable. \\ Competing interests \\ The authors declare that they have no competing interests.
}

Authors' contributions

The authors contributed equally to the writing of this paper. The authors read and approved the final manuscript.

\section{Author details}

${ }^{1}$ Department of Mathematics, Faculty of Art and Science, Kırklareli University, Kırklareli, Turkey. ${ }^{2}$ Department of Mathematics, Faculty of Art and Science, Giresun University, Giresun, Turkey.

\section{Publisher's Note}

Springer Nature remains neutral with regard to jurisdictional claims in published maps and institutional affiliations.

Received: 21 February 2019 Accepted: 9 July 2019 Published online: 17 July 2019

\section{References}

1. Alomari, M.W., Darus, M., Kirmaci, U.S.: Some inequalities of Hermite-Hadamard type for s-convex functions. Acta Math. Sci. Ser. B Engl. Ed. 31, 1643-1652 (2011)

2. Budak, H., Sarikaya, M.Z.: Some new generalized Hermite-Hadamard inequalities for generalized convex functions and applications. J. Math. Ext. 12(4), 51-66 (2018)

3. Dragomir, S.S.: Hermite-Hadamard inequalities for convex functions of selfadjoint operators in Hilbert spaces. Linear Algebra Appl. 436(5), 1503-1515 (2012)

4. Dragomir, S.S., Fitzpatrick, S.: The Hadamard inequalities for s-convex functions in the second sense. Demonstr. Math. 32(4), 687-696 (1999)

5. Dragomir, S.S., Pearce, C.E.M.: Selected Topics on Hermite-Hadamard Inequalities and Applications. Victoria University, Australia (2000)

6. Dragomir, S.S., Pečarić, J., Persson, L.E.: Some inequalities of Hadamard type. Soochow J. Math. 21, 335-341 (1995)

7. Hua, J., Xi, B.Y., Qi, F.: Hermite-Hadamard type inequalities for geometrically s-convex functions. Commun. Korean Math. Soc. 29(1), 51-63 (2014)

8. Hudzik, H., Maligranda, L.: Some remarks on s-convex functions. Aequ. Math. 48(1), 100-111 (1994)

9. İşcan, I.:. Hermite-Hadamard type inequalities for harmonically convex functions. Hacet. J. Math. Stat. 43(6), 935-942 (2014)

10. İşcan, İ.: New refinements for integral and sum forms of Hölder inequality (2019). Researchgate, Preprint, https://doi.org/10.13140/RG.2.2.19356.54409

11. Kadakal, M., Işcan, I., Kadakal, H., Bekar, K.: On improvements of some integral inequalities (2019). Researchgate, Preprint, https://doi.org/10.13140/RG.2.2.15052.46724

12. Kunt, M., Iş̧can, I.: Hermite-Hadamard-Fejer type inequalities for $p$-convex functions. Arab J. Math. Sci. 23, 215-230 (2017)

13. Latif, M.A.: Inequalities of Hermite-Hadamard type for functions whose derivatives in absolute value are convex with applications. Arab J. Math. Sci. 21, 84-97 (2015)

14. Latif, M.A., Dragomir, S.S., Momoniat, E.: On Hermite-Hadamard type integral inequalities for n-times differentiable $m$ and $(\alpha, m)$-logarithmically convex functions. Filomat 30(11), 3101-3114 (2016)

15. Liu, W., Wen, W., Park, J.: Hermite-Hadamard type inequalities for MT-convex functions via classical integrals and fractional integrals. J. Nonlinear Sci. Appl. 9, 766-777 (2016)

16. Mitrinovic, D.S., Pečarić, J., Fink, A.M.: Classical and New Inequalities in Analysis. Kluwer Academic, Dordrecth (1993)

17. Muddassar, M., Bhatti, M.I., lqbal, M.: Some new s-Hermite-Hadamard type inequalities for differentiable functions and their applications. Proc. Pak. Acad. Sci. 49(1), 9-17 (2012)

18. Pearce, C.E.M., Pečarić, J.: Inequalities for differentiable mappings with application to special means and quadrature formulae. Appl. Math. Lett. 13, 51-55 (2000)

19. Sarikaya, M.Z., Budak, H.: On generalized Hermite-Hadamard inequality for generalized convex function. Int. J. Nonlinear Anal. Appl. 8(2), 209-222 (2017) 
20. Shuang, Y., Qi, F.: Integral inequalities of Hermite-Hadamard type for extended $s$-convex functions and applications. Mathematics 6(11), Article ID 223 (2018)

21. Zhang, T.Y., Ji, A.P., Qi, F.: Some inequalities of Hermite-Hadamard type for GA-convex functions with applications to means. Le Mathematiche LXVII(I), 229-239 (2013)

Submit your manuscript to a SpringerOpen ${ }^{\mathcal{O}}$ journal and benefit from:

- Convenient online submission

$\checkmark$ Rigorous peer review

Open access: articles freely available online

- High visibility within the field

- Retaining the copyright to your article

Submit your next manuscript at $\gg$ springeropen.com 